\title{
Construction of a long non-coding RNA-mediated competitive endogenous RNA network reveals global patterns and regulatory markers in gestational diabetes
}

\author{
LEI LENG ${ }^{1}$, CHENGWEI ZHANG $^{1}$, LIHONG REN $^{2}$ and QIANG LI ${ }^{1}$ \\ ${ }^{1}$ Department of Endocrinology, The Second Affiliated Hospital of Harbin Medical University; \\ ${ }^{2}$ Department of Endocrinology, The Second Hospital of Harbin, Harbin, Heilongjiang 150006, P.R. China
}

Received August 8, 2018; Accepted November 27, 2018

DOI: $10.3892 / \mathrm{ijmm} .2018 .4026$

\begin{abstract}
Gestational diabetes mellitus (GDM) is a common disease affecting pregnant women. Recent studies have suggested that competing endogenous RNAs (ceRNAs), which compete with long non-coding RNAs (lncRNAs) for microRNA (miRNA or miR) binding and indirectly regulate miRNA targets through competing interactions, play a critical role in disease. In this study, we present a computationally integrated approach with which to construct a lncRNA-mediated ceRNA network (LCEN) in GDM by integrating RNA interactions and expression data. IncRNAs exhibited specific features and played critical roles in GDM-associated LCEN. The construction of a global functional score profile revealed that ceRNAs had a high activity in GDM. We extracted several ceRNA modules and demonstrated that these modules had increased close interactions. We further discovered that these ceRNA modules may be utilized as specific and effective circulating biomarkers for GDM. Finally, functional analyses demonstrated that the GDM-associated ceRNAs participated in the regulation of irisin and the thyroid hormone signaling pathway. It was suggested that there were close associations between the thyroid hormone and GDM. Collectively, ceRNAs may accelerate biomarker discovery and therapeutic development in GDM.
\end{abstract}

\section{Introduction}

Gestational diabetes mellitus (GDM) is defined as glucose intolerance with onset or first recognition during pregnancy and is one of the most common complications during pregnancy (1). Metabolic and immunological changes can

Correspondence to: Dr Qiang Li, Department of Endocrinology, The Second Affiliated Hospital of Harbin Medical University, 246 Xue Fu Road, Harbin, Heilongjiang 150006, P.R. China E-mail: harbinlq@126.com

Key words: long non-coding RNA, competitive endogenous RNA, gestational diabetes, topological feature, circulating biomarker occur during pregnancy and frequently present clinically as increased insulin resistance and immune tolerance against the fetus and placenta (2). The risk of adverse pregnancy outcomes is increased in patients with GDM and affects the mother and the child; common complications include ischemic heart disease, hypertension, predisposition to obesity, metabolic syndrome and type 2 diabetes mellitus (T2DM) following pregnancy (3). Risk factors include pre-pregnancy weight gain and obesity, a family history of diabetes, an advanced maternal age, a poor diet and low physical activity $(4,5)$. In addition, studies have reported that low thyroid hormone levels during early pregnancy are associated with an increased risk of GDM and may affect pregnancy outcome and the intellectual level of the infant $(6,7)$. However, the etiology of and mechanisms responsible for GDM are unknown for the majority of patients and thus, the identification of novel signatures or biomarkers that enhance clinical behavior in the treatment of GDM are essential.

Long non-coding RNAs (lncRNAs) do not code for proteins and are pervasive across the genome (8). The dysregulation of lncRNA expression is associated with various human diseases (9-11). A previous study suggested metastasis-associated lung adenocarcinoma transcript 1 (MALAT1) lncRNA expression may be a novel biomarker to predict GDM (12). Shi et al (13) performed a microarray expression profile analysis to reveal that IncRNAs were differentially expressed in the blood of the umbilical cord from patients with GDM and may play a role in macrosomia development. Recently, an increasing number of studies have suggested that lncRNAs participate in competing endogenous RNA (ceRNA) regulatory processes and in communicating with other RNAs, such as microRNAs (miRNAs or miRs), coding genes and circular RNAs (circRNAs) $(14,15)$. IncRNAs compete with miRNA target genes for binding by sharing common miRNA-binding sites, thus attenuating miRNA-associated target repression. UICLM lncRNA has been shown to promote liver metastasis in colorectal cancer by acting as a ceRNA for miR-215, regulating zinc finger E-box-binding homeobox 2 (ZEB2) expression (16). MT1JP lncRNA has been shown to function as a ceRNA in regulating FBXW7 by competitively binding to miR-92a-3p in gastric cancer (17). In addition, studies have utilized high-throughput expression profiles and interaction 
data to construct a global ceRNA network in diseases $(18,19)$. These data highlight the important role of lncRNA in interacting with ceRNAs and suggest that the integration of expression profiles and network analysis contributes to the identification of problematic lncRNAs and to the elucidation of the mechanisms of disease. However, a lncRNA-mediated ceRNA network in GDM has not yet been constructed and analyzed, at least to the best of our knowledge.

In this study, we used a comprehensive computational approach to construct a lncRNA-mediated ceRNA network (LCEN) in GDM by integrating the expression profiles of mRNAs, miRNAs and lncRNAs from patients with GDM and experimentally verifying the interactions. We observed that the lncRNAs exhibited specific topological features in the LCEN, consistent with a regulatory association with coding mRNAs in GDM. The LCEN in GDM presents specific and highly competing activity profiles. We further identified a core GDM-associated subnetwork and 3 modules to characterize the properties of LCENs. ceRNA expression in these modules may be able to distinguish between patients that are normal glucose-tolerant (NGT) and patients with GDM. Functional analysis revealed that GDM-associated ceRNAs participated in glycolytic and hormone metabolic processes. Additionally, we identified the thyroid hormone pathway to be associated with ceRNAs in GDM. On the whole, these results suggest that GDM-associated LCENs may provide new insight into the mechanisms of GDM and may aid in the discovery of novel molecular biomarkers and GDM therapeutic strategies.

\section{Materials and methods}

Collection of high-throughput data for IncRNAs, miRNAs and genes. IncRNA, miRNA and gene expression profiles for GDM were downloaded from the Gene Expression Omnibus database (www.ncbi.nlm.nih.gov/geo). The study collected data for 8 NGT patients and 8 patients with GDM, which were matched by body mass index and age. Paired data were adjusted for mid-pregnancy weight gain and by pregnancy week (accession no. GSE92772; unpublished data). The RNA sequencing of whole blood cells of these samples were produced by Illumina HiSeq 2500.

Experimental validation of miRNA targets and GDM-associated genes. Gene-miRNA association data were obtained from a public database (miRTarBase 7.0) that contained >360,000 miRNA-target interactions (MTIs) (20). Only MTIs supported by strong experimental evidence were extracted for the current study. IncRNA-miRNA association data was obtained (RAID 2.0) containing experimentally and computationally predicted RNA-RNA and RNA-protein interactions. We further extracted IncRNA-miRNA association data supported by strong experimental evidence (21). GDM-associated gene data were downloaded from DisGeNET, a discovery platform containing one of the largest publicly available collections of genes and variants associated with human diseases (22).

Identification of candidate ceRNA interactions. A hypergeometric test was performed to identify candidate competing mRNA-lncRNA interactions. We evaluated the significance of shared miRNAs between lncRNAs and mRNAs. The human genome contains a total number of $\mathrm{K}$ miRNAs, with $\mathrm{L}$ and $\mathrm{M}$ representing the number of miRNAs associated with the current lncRNA or mRNA, respectively. The number of common miRNAs shared by IncRNA and mRNA was defined as ' $\mathrm{i}$ '. P-values were calculated using the hypergeometric test and by evaluating the enrichment significance for competing function as follows:

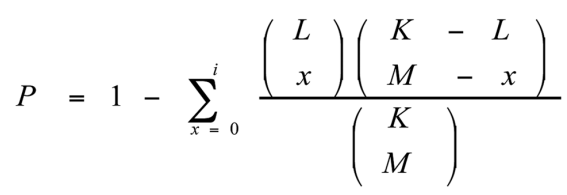

A false discovery rate (FDR) correction was applied. FDR $<0.01$ was the threshold to select candidate competing mRNA-IncRNA pairs.

Identification of GDM-associated functional ceRNAs by integrating expression and candidate ceRNA interactions. Pearson correlation coefficients (PCC) were used to identify GDM-specific mRNA-lncRNA pairs based on the expression values of competing mRNA-lncRNA pairs. We filtered GDM-associated functional ceRNAs from expression datasets based on following rules: PCC (lncRNA, gene) $>0.5$ and $\mathrm{P}<0.05$; PCC (IncRNA, miRNA) $<-0.5$ and $\mathrm{P}<0.05$; and $\mathrm{PCC}$ (gene, miRNA) $<-0.5$ and $\mathrm{P}<0.05$, with PCC representing interactions based on expression values. A total of 77 GDM-associated functional ceRNAs, comprising 17 lncRNAs, 14 miRNAs and 40 coding genes, were retained for further analysis. A GDM-associated ceRNA network was constructed using Cytoscape 3.0 (http://www.cytoscape.org/).

Functional score of GDM-associated functional ceRNAs. The correlation of mRNA and miRNA expression is an effective statistically method for distinguishing between direct and indirect interactions (23). The functional score was applied to determine the strength of competition in GDM-associated functional ceRNAs and was defined as (IPCC (IncRNA, gene)|+|PCC (lncRNA, miRNA)|+|PCC (gene, miRNA)|)/3. A higher functional score indicated a greater competition between lncRNA and gene for miRNA binding.

Dissecting topological features for LCEN in GDM. We performed topological analyses, including degree and topological coefficients for all the nodes in LCEN using Cytoscape 3.0 (http://www.cytoscape.org/).

Identifying core modules from subnetworks of LCEN in GDM. We extracted a subnetwork form LCEN in GDM following the verification GDM-associated genes. All the network modules were identified using ClusterONE with the default parameters based on the subnetwork of LCEN constructed by GDM-associated genes (http://apps.cytoscape. org/apps/ClusterONE). ClusterONE is a tool that clusters a given network based on topology to identify densely connected regions. A total of 14 modules were identified and the 3 modules with the highest numbers of nodes were extracted.

Classification power of the core modules in GDM. For core modules, the expression data was used to classify 
A
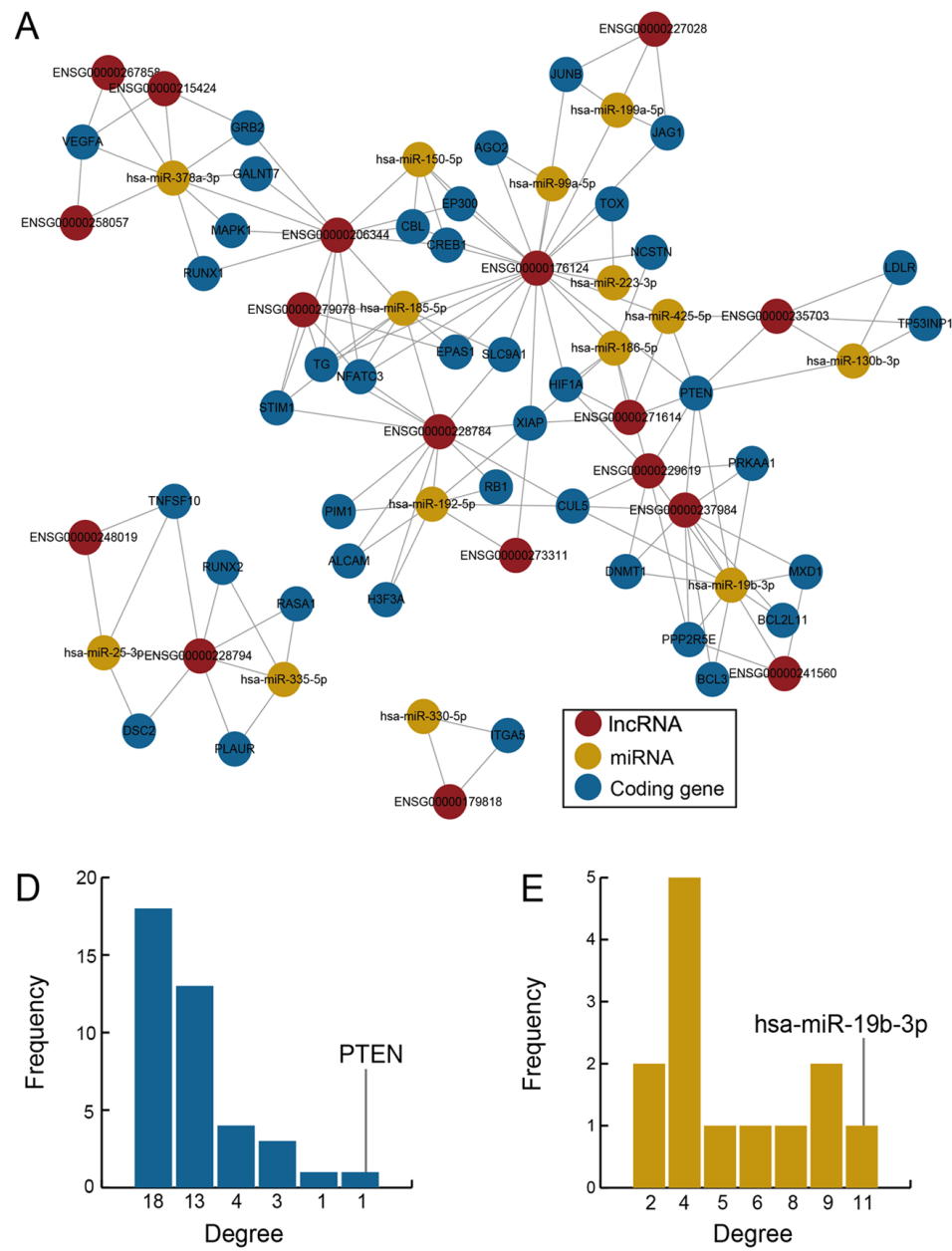

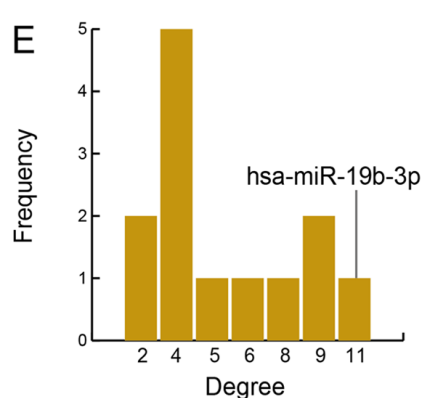

B

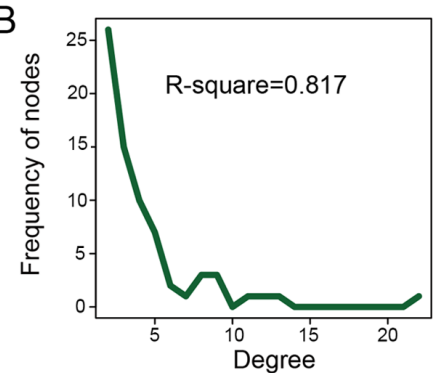

C
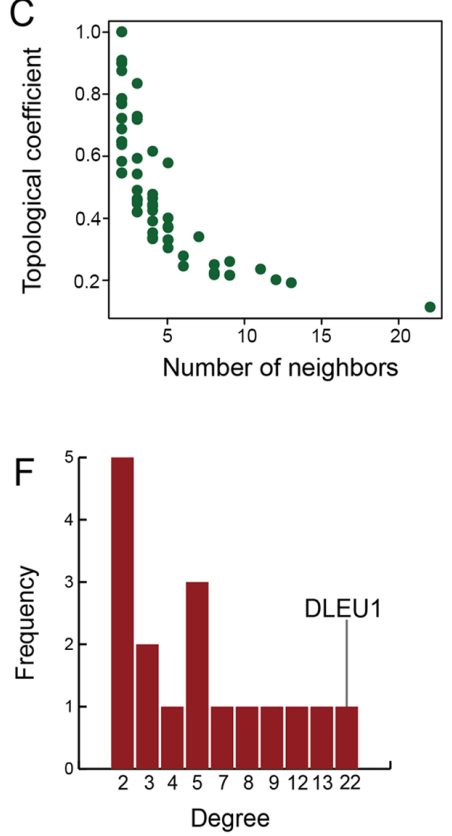

Figure 1. Construction and global characteristics of the LCEN in GDM. (A) A global LCEN in GDM. Coding genes, lncRNAs and miRNAs are colored blue, red and yellow, respectively. (B) Degree distribution of nodes in the LCEN. (C) Topological coefficient of nodes in the LCEN. The degree distributions of (D) genes, (E) miRNAs and (F) lncRNAs; the horizontal axis represents the degree values and vertical axis represents the number of nodes with a certain degree value. LCEN, IncRNA-mediated ceRNA network; GDM, gestational diabetes mellitus; lncRNA, long non-coding RNA; miRNA, microRNA; ceRNA, competitive endogenous RNA.

16 samples (NGT, $\mathrm{n}=8$ and GDM, $\mathrm{n}=8$ ) applying a consensus clustering method (24). This process is performed by ConsensusClusterPlus package in R (https://www.r-project. org/). We defined the optimal category number as the smallest increase in the area under the cumulative distribution function (CDF) curve. Chi-square tests were used to evaluate whether the disease and control samples can be classified using this method. A value of $\mathrm{P}<0.05$ was considered to indicate a statistically significant difference. Gene, miRNA and lncRNA expression were used to perform this analysis and the respective expression was integrated.

Functional enrichment analysis. With the online Enrichr tool and by applying default parameters, a functional enrichment analysis was performed for genes in LCEN (25). We identified enriched GO terms $(\mathrm{P}<0.01)$ and KEGG pathways $(\mathrm{P}<0.05)$.

\section{Results}

Construction and global properties of the GDM-associated ceRNA network. A total of 480,023 candidate ceRNA interactions (gene-miRNA-lncRNA) were identified. An integrated pipeline was used to construct a GDM-associated LCEN based on experimentally verified RNA interactions and expression data (Fig. 1A). The network consisted of 71 nodes (17 lncRNAs, 14 miRNAs and 40 coding genes) and 150 edges. A total of 76 gene-lncRNA interactions, 30 miRNA-lncRNA interactions and 44 gene-miRNA interactions were identified in the GDM-associated LCEN. The GDM-associated LCEN exhibited a scale-free distribution $\left(\mathrm{R}^{2}=0.817\right)$ and was similar to the majority of biological networks (Fig. 1B). It was further indicated that this network had a similarity to the small-world network (26). In addition, it was discovered that the topological coefficient decreased when the degree increased, which suggested that the GDM-associated LCEN described a hierarchical modularity phenomenon (Fig. 1C) (27). This topological feature has been presented in ceRNA networks of various types of cancer (19). Furthermore, we determined the degrees of coding genes, miRNAs and lncRNAs. A node was considered as a hub, with increased ceRNA interactions representing higher node degrees. In the current study, lncRNA nodes exhibited higher mean degrees compared with coding gene and miRNA nodes (Fig. 1D-F). The mean degree of coding for genes, miRNAs and lncRNAs was 3, 5.54 and 9.40, respectively. These results suggested that although lncRNA do not code for proteins, they exhibited more specific topological 

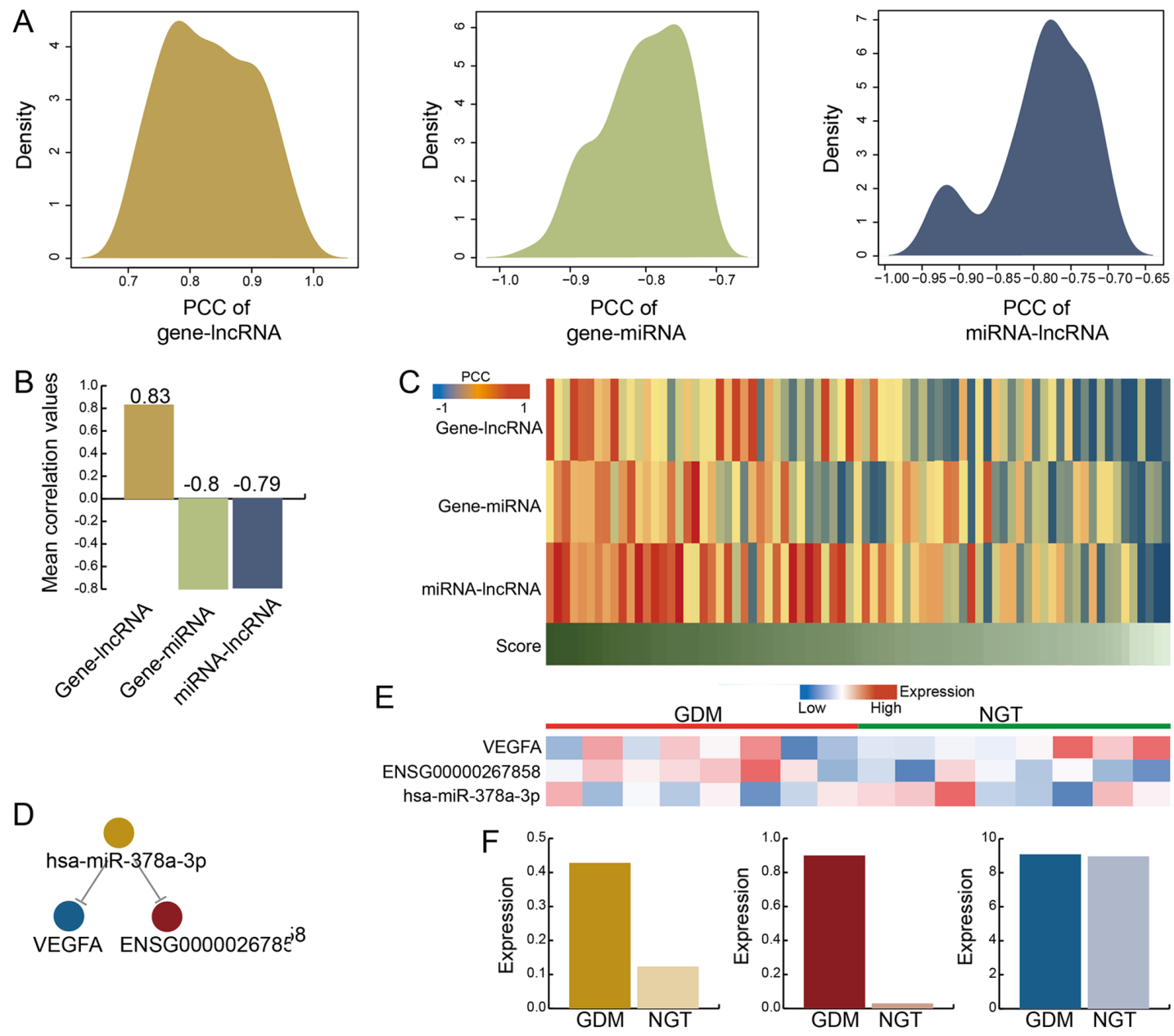

Figure 2. Construction of a functional score profile for the GDM-associated LCEN. (A) Density distribution curves of gene-lncRNA, gene-miRNA and miRNA-lncRNA. (B) Mean correlation values of gene-lncRNA, gene-miRNA and miRNA-lncRNA interactions. (C) Functional score profile for functional ceRNAs in GDM. (D) An exemplary ceRNA. (E) Heatmap of ceRNA expression in GDM and NGT. (F) Mean expression values in GDM and NGT. GDM, gestational diabetes mellitus; LCEN, IncRNA-mediated ceRNA network; lncRNA, long non-coding RNA; miRNA, microRNA; ceRNA, competitive endogenous RNA; NGT, normal glucose-tolerant.

properties than mRNAs in the GDM-associated LCEN. The highest degree nodes were determined for phosphatase and tensin homolog (PTEN), hsa-miR-19b-3p and deleted in lymphocytic leukemia 1 (DLEU1).

Variable competing activity profiles in the GDM-associated ceRNA network. To characterize the compactness of each ceRNA interaction in the GDM-associated LCEN, we evaluated the PCC values for the gene-lncRNA, miRNA-lncRNA and miRNA-gene interactions. Density curves for these interactions were determined and observed to be similar (Fig. 2A). The majority PCC values were concentrated between 0.75-0.80 and the results indicated that interactions were compact. The mean PCC value of gene-lncRNA interactions was highest with 0.83 and the miRNA-gene and miRNA-lncRNA interactions had mean PCC values of -0.8 and -0.79 , respectively (Fig. 2B). In addition, a functional score was proposed to evaluate the GDM-associated functional ceRNAs. Based on the functional score, we constructed a competing activity profile for the GDM-associated LCEN (Fig. 2C). We discovered that while certain interactions exhibited lower PCC values, the overall ceRNAs exhibited a high functional score. The result indicated that the functional score may be used to evaluate the properties of ceRNA interactions. We further discovered that the gene expression for VEGFA competed with IncRNA expression of MZF1-AS1 and hsa-miR-378a-3p expression (Fig. 2D). The expression heatmap exhibited differences between GDM and NGT (Fig. 2E). The expression levels of VEGFA, MZF1-AS1 and hsa-miR-378a-3p in GDM were increased compared with NGT (Fig. 2F). We further observed that the difference in lncRNA levels between GDM and NGT was not significant, potentially due to decreased lncRNA expression in blood. The results suggested that gene and miRNA associations with ceRNA may potentially be used to explore the role and function of IncRNA, especially in blood sample.

Characterization of a core GDM-associated subnetwork. We extracted a subnetwork based on verified GDM-associated genes to explore how ceRNAs provide insight into GDM pathogenesis. We observed that $57.5 \%$ of the genes in the GDM-associated LCEN were verified as GDM-associated genes (Fig. 3A). We further counted the degree of nodes in the subnetwork (Fig. 3B). DLEU1 lncRNA exhibited the highest degree, which indicated that it may play an essential role in the GDM-associated LCEN. The subnetwork consisted of 50 nodes ( 23 coding genes, 12 miRNAs and 15 lncRNAs) 
A

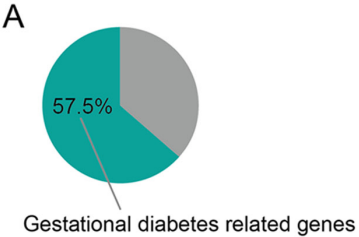

B 15

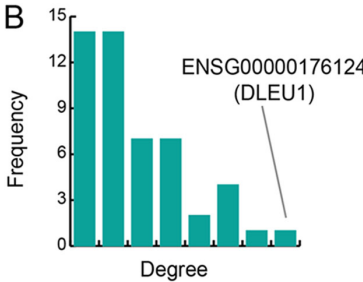

D

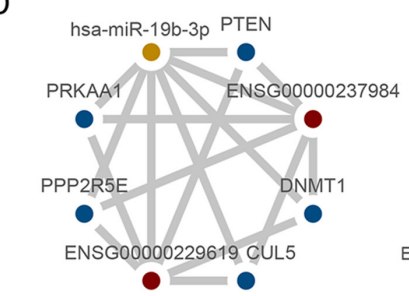

C
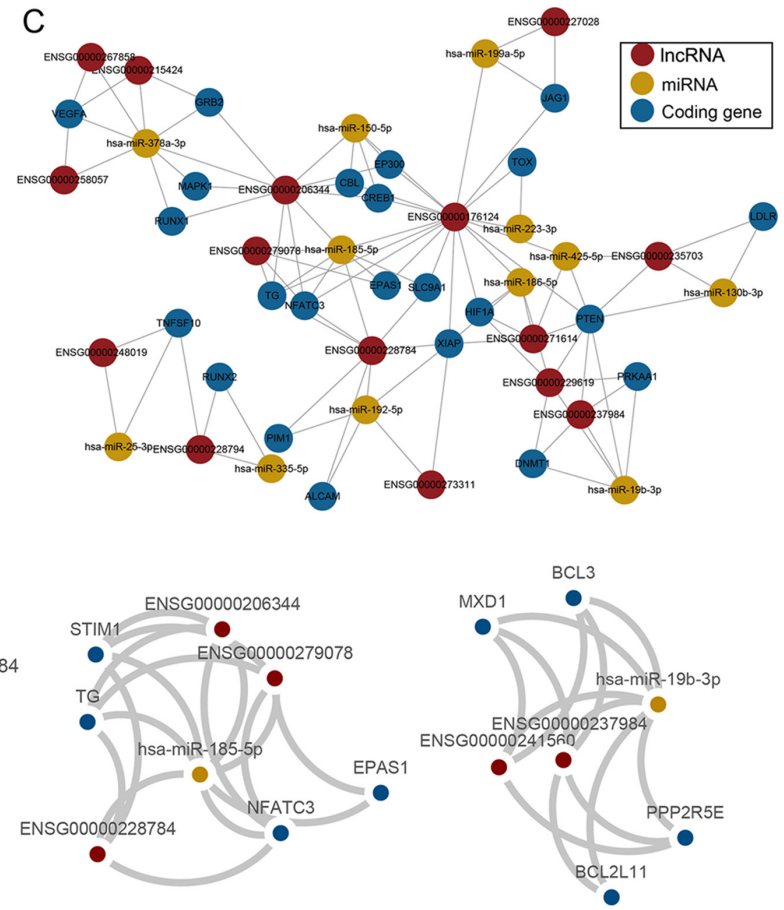

$\mathrm{E}$

Positive correlation

Negative correlation Score

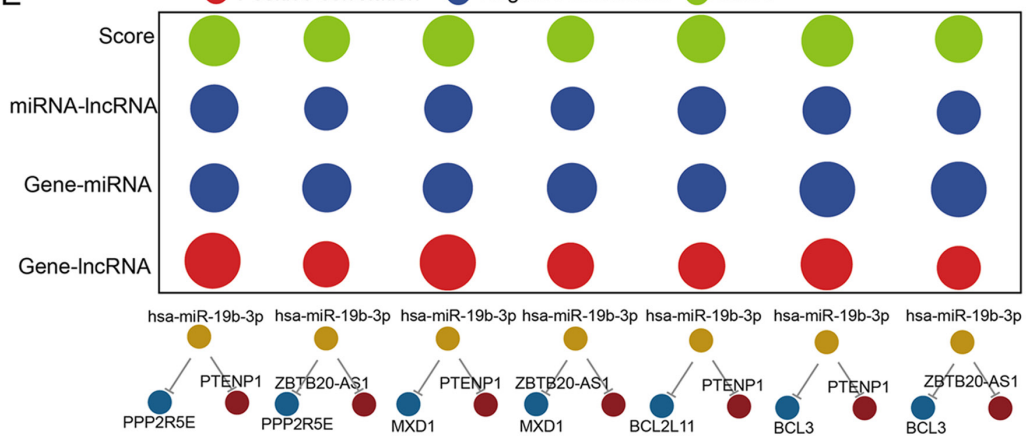

Figure 3. Core subnetwork and ceRNA modules in GDM. (A) Percentage of known GDM-associated genes in all genes. (B) Degree of nodes in the subnetwork (C) A subnetwork extracted from LCEN, which only included known GDM-associated genes and interacting RNAs. (D) Three ceRNA modules extracted from the subnetwork. (E) Co-expression pattern of each ceRNA in the third module; circle size reflects correlation strength; red represents a positive and blue a negative correlation; the functional score is presented in green. ceRNA, competitive endogenous RNA; GDM, gestational diabetes mellitus.

and 103 edges (Fig. 3C). A module analysis of the subnetwork was performed to further investigate the interactions between various RNA transcripts. Three modules were extracted, and the number of nodes and features were analyzed (Fig. 3D). The first module included 8 nodes (5 coding genes, 1 miRNA and 2 lncRNAs); the nodes formed 10 ceRNAs. The second module included 8 nodes ( 4 coding genes, 1 miRNA and 3 lncRNAs); the nodes formed 10 ceRNAs. The third module included 7 nodes ( 4 coding genes, 1 miRNA and 2 lncRNAs); the nodes formed 7 ceRNAs. A small quantity of nodes formed multiple ceRNAs indicating these nodes had strong associations. We further considered the compactness of each ceRNA interaction in modules and discovered the ceRNA interactions in modules were more compact. In the third module, all 7 ceRNAs had high functional scores (Fig. 3E) suggesting that the nodes in this module were close.

GDM-associated ceRNA distinguish patients with NGT and GDM. To evaluate whether ceRNAs are potential classifiers in GDM, we used gene, miRNA and lncRNA expression values from each module to classify the samples using a consensus clustering method. We further used a Chi-square test to validate the classification power of the modules. No significant differences in gene, miRNA or lncRNA expression between GDM and NGT samples were observed in these 3 modules; however, significance was determined for the integration of first and second module ( $\mathrm{P}=0.03$; Fig. $4 \mathrm{~A})$. The results indicated that ceRNAs may be an effective classifier for patients with GDM. We then analyzed the classification power to evaluate the integrated expression of 9 coding genes [cullin 5 (CUL5), DNA methyltransferase 1 (DNMT1), endothelial PAS domain protein 1 (EPAS1), nuclear factor of activated T cells 3 (NFATC3), protein phosphatase 2 regulatory subunit B' epsilon (PPP2R5E), protein kinase AMP-activated catalytic subunit alpha 1 (PRKAA1), PTEN, stromal interaction molecule 1 (STIM1) and thyroglobulin (TG)], 5 lncRNAs (HCG27, LINC00954, MBNL1-AS1, PTENP1 and SND1-IT1) and 2 miRNAs (miR-19b-3p and miR-185-5p). The GDM and NGT samples could be classified into several groups by the above-mentioned genes, lncRNAs and miRNAs. According to the $\mathrm{CDF}$ and relative change in the area under the curve (Fig. 4C and D), we determined that the final number of groups 

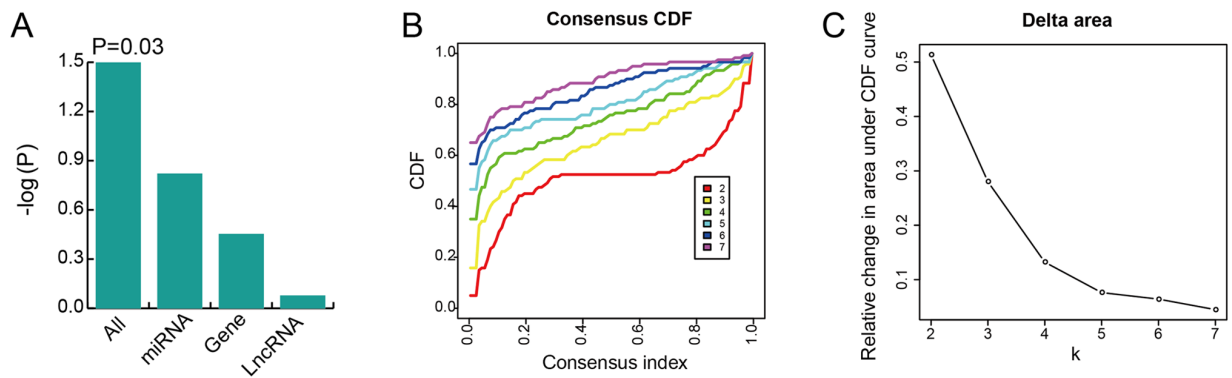

$\mathrm{D}$
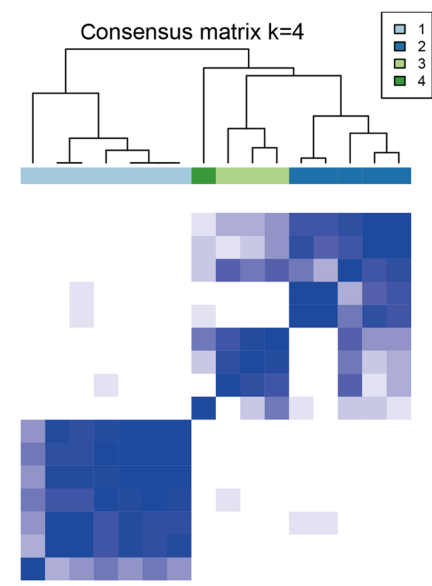

E

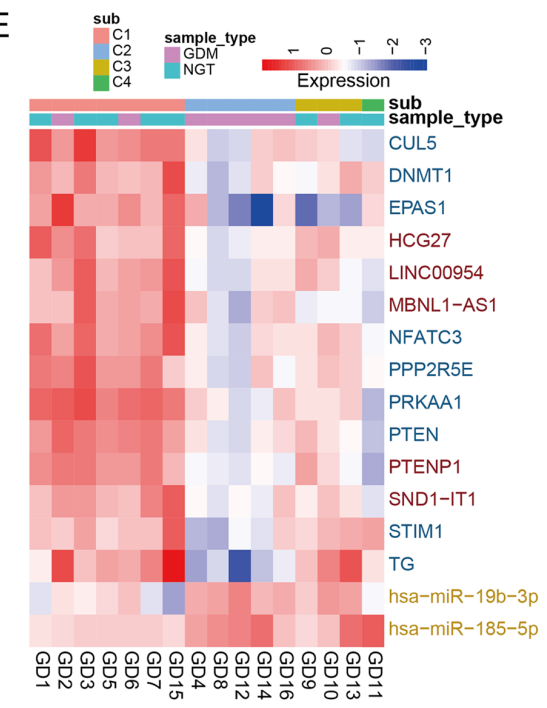

Figure 4. ceRNA modules distinguish NGT and GDM samples. (A) P-values from the Fisher-test classifying the power of miRNA, genes and lncRNA. (B) Cumulative distribution function of the consensus index. (C) Relative changes in the area under the CDF curve by group number. (D) Consensus cluster heatmap of samples. (E) Gene expression heatmap, with sublabels referring to group type classified by the consensus cluster method and the sample type referring to disease status of samples. The names of lncRNAs, genes and miRNAs are represented by blue, red and yellow. ceRNA, competitive endogenous RNA; GDM, gestational diabetes mellitus; NGT, normal glucose-tolerant; miRNA, microRNA; lncRNA, long non-coding RNA.

was 4 . We discovered that the 4 sample groups had respective expression patterns (Fig. 4D). We further determined that a majority of samples was classified accurately, particularly in the second group (C2), where all samples were from GDM (Fig. 4E). Collectively, these results suggested that the integration of expression in ceRNA interactions can distinguish between NGT and GDM samples.

GDM-associated ceRNA is associated with critical biological functions and the thyroid hormone. We performed GO enrichment analysis based on the genes in the GDM-associated LCEN. The enrichment of these genes in different GO terms was observed (Fig. 5A). Genes were associated with some critical biological functions, including the positive regulation of gene expression and the positive regulation of transcription. Certain genes exhibited metabolism-associated functions, such as the enrichment of the regulation of macromolecular, carbohydrate and ATP processes. In addition, we discovered associations to glycolytic processes. Significantly enriched GO functions were associated with hormones, including intracellular steroid production and hormone metabolism. We further performed pathway enrichment analysis and identified insulin-associated pathways to be relevant in the GDM-associated LCEN (Fig. 5B). The thyroid hormone signaling pathway was identified in the analysis and this hormone may be a potential biochemical marker in predicting GDM and effects on insulin sensitivity in $\operatorname{GDM}(28,29)$. As the thyroid hormone status has previously been demonstrated to be associated with GDM during pregnancy $(29,30)$, we further analyzed this signaling pathway and identified 4 enriched genes (Fig. 5C). These genes participated in 8 ceRNA interactions and the pathway was identified to be associated with the gluconeogenesis pathway, which is an essential pathway in diabetes (31). We further focused on irisin, which is an exercise-induced myokine that drives brown-fat-type thermogenesis in murine white fat (32). In this analysis, we observed associations with the MAPK signaling pathway and importance arises from irisin potentially suppressing obesity and associated T2DM via the MAPK signaling pathway (33). A ceRNA module identified in the current study, MAPK1/miR-378/HCG27, may be associated with irisin based to literature known MAPK1 and miR-378 associations with irisin (34,35). Additionally, hyper- and hypothyroidism are associated with the upregulation of serum irisin in male rats (36). These results suggested GDM-associated ceRNAs may participate in the regulation of the thyroid hormone and irisin and influence GDM development.

\section{Discussion}

Coding genes, miRNAs and lncRNAs can form complex regulatory clusters, including ceRNAs, which may influence pathogenic mechanisms in GDM. lncRNAs regulate mRNA expression through an indirect post-transcriptional 
A

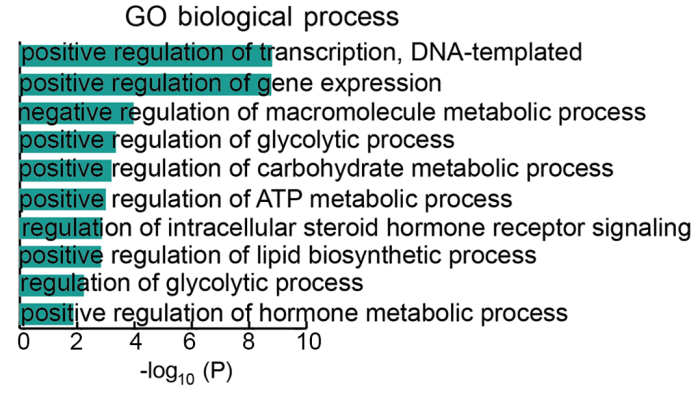

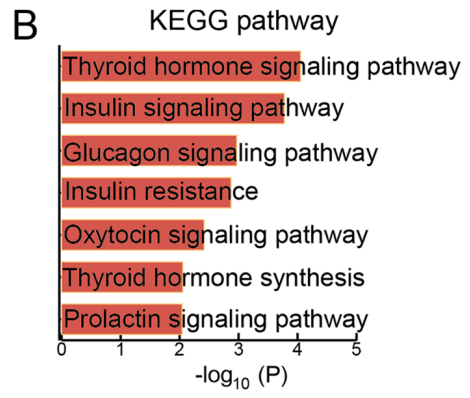

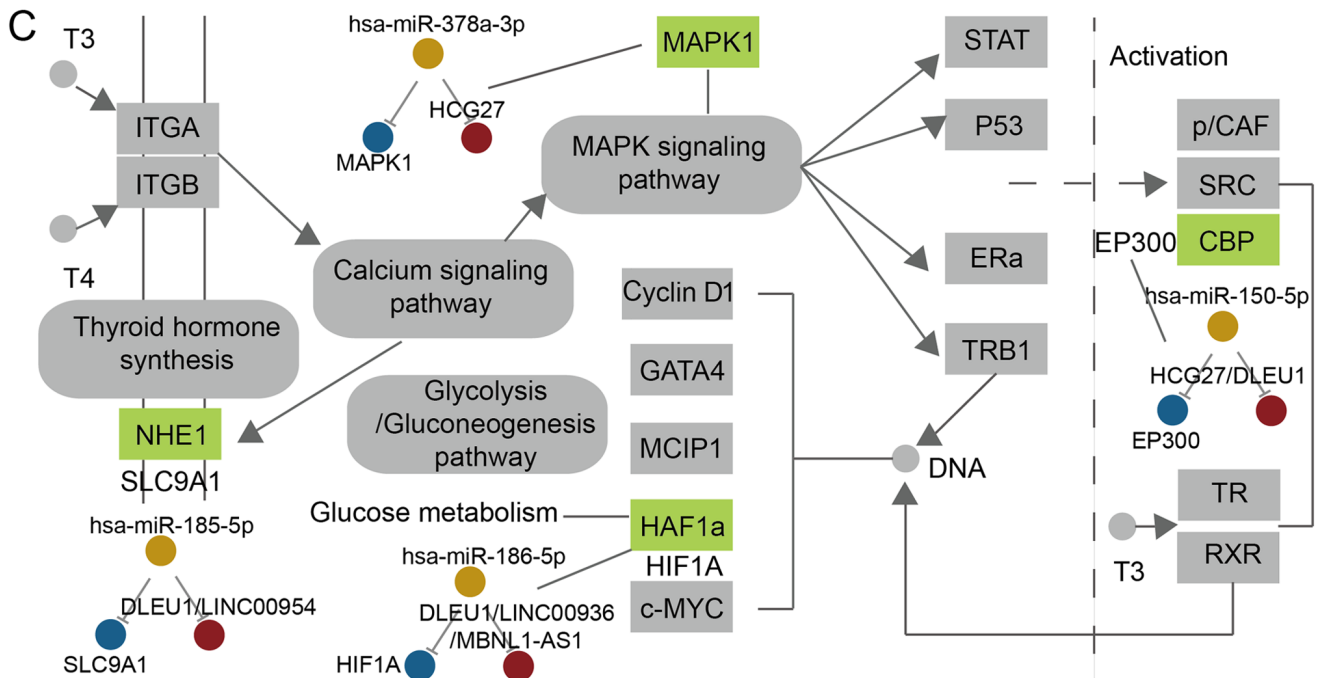

Figure 5. Functional analysis of genes in the GDM-associated LCEN. (A) Enriched GO terms for genes in the GDM-associated LCEN ranked by -log10(P). (B) KEGG pathway enrichment for genes in the GDM-associated LCEN, ranked by $-\log 10(\mathrm{P})$. (C) Association of the thyroid hormone signaling pathway with GDM-associated ceRNAs. Some dysregulated ceRNAs are showed in figure. GDM, gestational diabetes mellitus; LCEN, lncRNA-mediated ceRNA network; ceRNA, competitive endogenous RNA.

mechanism of competing with miRNA for mRNAs binding sites (14). In various diseases, individual ceRNA crosstalk interactions were verified and global ceRNA networks were constructed (37-39). Lin et al (40) constructed a ceRNA network to reveal the regulatory role of lncRNAs in T2DM. However, Lin et al (40) solely relied on disease-associated genes to construct ceRNA network and expression information was not integrated. The lack of expression information reduced specificity and accuracy in the disease biomarker analysis. In this study, a system was created that integrated experimentally-verified RNA interactions, expression data and verified GDM-associated genes. A global ceRNA network was constructed and GDM-associated ceRNAs were identified. A candidate GDM-associated ceRNA list were provided and some lncRNAs, genes and miRNAs in this list have not been verified by strong evidence. In future studies, researchers could extracted these molecules to construct relations between non-coding RNAs and GDM or identify more accurate biomakers and treatment targets for GDM. Collectively, the current study provides a comprehensive resource for studying the regulation of GDM by non-coding molecules.

Various lncRNAs function as molecular biomarkers of different types of diseases. IncRNA-HEIH levels in serum and exosomes are potential biomarkers for $\mathrm{HCV}$-associated hepatocellular carcinoma (41). Plasma lncRNA GAS5 is a novel biomarker for coronary artery disease (42). Survival analyses further demonstrated that ceRNA network modules are potential prognostic biomarkers in GBM (43). In the current analysis, we found that although single lncRNAs could not perfectly distinguish GDM and NGT (Chi-square test, $\mathrm{P}>0.05$ ), some combinations among lncRNAs, genes and miRNAs could distinguish GDM and NGT (Chi-square test, $\mathrm{P}=0.03$ ). The result also verified the advantage of considering the ceRNA as specific and effective biomarkers to distinguish between NGT and GDM. We inferred that IncRNAs could interact with other molecules (including miRNAs and genes) to play their roles in some diseases. Blood-based biomarkers are critical for disease prediction. In this study, it was suggested that certain candidate ceRNAs in the blood may serve as circulating biomarkers in GDM and more experiments focused on whole ceRNA motif in cell lines or animal models should be constructed.

The frequency of thyroid dysfunction in diabetic patients is increased compared with the general population and $\leq 1 / 3$ of patients with type 1 diabetes develop thyroid dysfunction (44). Thyroid hormones are positively associated with insulin resistance in the early development of T2DM (45). However, little attention has been paid to the diagnosis of thyroid diseases in diabetics, as they are diagnosed in only approximately half of the patients (46). In the current study, we discovered various GDM-associated ceRNAs associated with the thyroid hormone signaling pathway. For example, SLC9A1 is a key upstream gene of thyroid hormone signaling pathway and form two dysregulated ceRNAs with miR-185-5p 
and lncRNA DLEU1 and LINC00954 in GDM. DLEU1 was associated with BMI-adjusted adiponectin, which is related to diabetes (47). In this study, IncRNA DLEU1 had the highest degree in GDM-associated subnetwork (Fig. 3B). We provide novel insight into how IncRNA DLEU1 influences thyroid hormone synthesis by competing miR-185-5p with SLC9A1. The MAPK signaling pathway is an essential part of the thyroid hormone signaling pathway (34). In this study, we found that lncRNA HCG27 competes for miR-378a-3p with MAPK1 in GDM, and MAPK1 and miR-378 have both been shown to be associated with irisin in previous studies $(34,35)$. Irisin is expressed and produced by human muscle and adipose tissue in association with obesity and insulin resistance (48). Thus, we inferred that there were close relations among irisin, thyroid hormone, obesity and GDM. In summary, novel insight was provided into the study of the associations between thyroid hormone dyscrasia and GDM. Further studies are warranted to focus on investigating an increased number of GDM samples to validate the accuracy and stability of the method presented in the current study.

In conclusion, in the present study, we constructed a GDM-associated LCEN and analyzed its features. A functional score was used to evaluate the activity of each ceRNA in GDM and certain ceRNA modules were extracted analyzed for strict interactions. In addition, these ceRNA modules demonstrated to distinguish between GDM and NGT samples. We further observed a close association between thyroid hormone dyscrasia and GDM. The current analysis provides insight into the identification of novel biomarkers for GDM.

\section{Acknowledgements}

Not applicable.

\section{Funding}

The current study was supported by the National Health and Family Planning Commission Public Welfare Industry Special (grant no. 201502007).

\section{Availability of data and materials}

All data generated or analyzed during this study are included in this published article.

\section{Authors' contributions}

LL and QL conceived and designed the present study. LL and $\mathrm{CZ}$ performed the experiments and analyzed the data. LR validated and improved the computational approach in this study. LL and LR wrote the manuscript. All authors read and approved the final manuscript.

\section{Ethics approval and consent to participate}

Not applicable.

\section{Patient consent for publication}

Not applicable.

\section{Competing interests}

The authors declare that they have no competing interests.

\section{References}

1. Crusell MKW, Hansen TH, Nielsen T, Allin KH, Rühlemann MC, Damm P, Vestergaard H,Rørbye C,Jørgensen NR, Christiansen OB, et al: Gestational diabetes is associated with change in the gut microbiota composition in third trimester of pregnancy and postpartum. Microbiome 6: 89, 2018.

2. Zhang C, Bao W, Rong Y, Yang H, Bowers K, Yeung E and Kiely M: Genetic variants and the risk of gestational diabetes mellitus: a systematic review. Hum Reprod Update 19: 376-390, 2013.

3. Daly B, Toulis KA, Thomas N, Gokhale K, Martin J, Webber J, Keerthy D, Jolly K, Saravanan P and Nirantharakumar K: Increased risk of ischemic heart disease, hypertension, and type 2 diabetes in women with previous gestational diabetes mellitus, a target group in general practice for preventive interventions: a population-based cohort study. PLoS Med 15: e1002488, 2018

4. Bellou V, Belbasis L, Tzoulaki I and Evangelou E: Risk factors for type 2 diabetes mellitus: an exposure-wide umbrella review of meta-analyses. PLoS One 13: e0194127, 2018.

5. Zhang $\mathrm{C}$ and Ning Y: Effect of dietary and lifestyle factors on the risk of gestational diabetes: Review of epidemiologic evidence. Am J Clin Nutr 94 (Suppl 6): 1975S-1979S, 2011.

6. Xu C and Zhang Z: Comparative study of thyroid hormone and antithyroid antibody levels in patients with gestational diabetes mellitus and pregnant patients with diabetes. Minerva Endocrinol 43: 126-130, 2018.

7. Yang S, Shi FT, Leung PC, Huang HF and Fan J: Low thyroid hormone in early pregnancy is associated with an increased risk of gestational diabetes mellitus. J Clin Endocrinol Metab 101: 4237-4243, 2016.

8. St Laurent G, Wahlestedt C and Kapranov P: The Landscape of long noncoding RNA classification. Trends Genet 31: 239-251, 2015.

9. Zhang M, Gu H, Xu W and Zhou X: Down-regulation of lncRNA MALAT1 reduces cardiomyocyte apoptosis and improves left ventricular function in diabetic rats. Int J Cardiol 203: 214-216, 2016.

10. Qin J, Bao H and Li H: Correlation of long non-coding RNA taurine-upregulated gene 1 with disease conditions and prognosis, as well as its effect on cell activities in acute myeloid leukemia. Cancer Biomark: Nov 4, 2018 (Epub ahead of print).

11. Shu J, Li S, Chen YB, Zhu QF and Yu XH: Long non-coding RNA EPB41L4A-AS2 inhibited non-small cell lung cancer proliferation, invasion and promoted cell apoptosis. Neoplasma 65: 664-672, 2018.

12. Zhang Y, Wu H, Wang F, Ye M, Zhu $\mathrm{H}$ and Bu S: Long non-coding RNA MALAT1 expression in patients with gestational diabetes mellitus. Int J Gynaecol Obstet 140: 164-169, 2018.

13. Shi Z, Zhao C, Long W, Ding H and Shen R: Microarray expression profile analysis of long non-coding RNAs in umbilical cord plasma reveals their potential role in gestational diabetes-induced macrosomia. Cell Physiol Biochem 36: 542-554, 2015.

14. Salmena L, Poliseno L, Tay Y, Kats L and Pandolfi PP: A ceRNA hypothesis: the Rosetta Stone of a hidden RNA language? Cell 146: 353-358, 2011.

15. Ebert MS and Sharp PA: Emerging roles for natural microRNA sponges. Curr Biol 20: R858-R861, 2010.

16. Chen DL, Lu YX, Zhang JX, Wei XL, Wang F, Zeng ZL, Pan ZZ, Yuan YF, Wang FH, Pelicano H, et al: Long non-coding RNA UICLM promotes colorectal cancer liver metastasis by acting as a ceRNA for microRNA-215 to regulate ZEB2 expression. Theranostics 7: 4836-4849, 2017.

17. Zhang G, Li S, Lu J, Ge Y, Wang Q, Ma G, Zhao Q, Wu D, Gong W, Du M, et al: LncRNA MT1JP functions as a ceRNA in regulating FBXW7 through competitively binding to miR-92a-3p in gastric cancer. Mol Cancer 17: 87, 2018.

18. Xu J, Feng L, Han Z, Li Y, Wu A, Shao T, Ding N, Li L, Deng W, Di X, et al: Extensive ceRNA-ceRNA interaction networks mediated by miRNAs regulate development in multiple rhesus tissues. Nucleic Acids Res 44: 9438-9451, 2016. 
19. Wang P, Ning S, Zhang Y, Li R, Ye J, Zhao Z, Zhi H, Wang T, Guo Z and Li X: Identification of lncRNA-associated competing triplets reveals global patterns and prognostic markers for cancer. Nucleic Acids Res 43: 3478-3489, 2015.

20. Chou CH, Shrestha S, Yang CD, Chang NW, Lin YL, Liao KW, Huang WC, Sun TH, Tu SJ, Lee WH, et al: miRTarBase update 2018: a resource for experimentally validated microRNA-target interactions. Nucleic Acids Res 46 (D1): D296-D302, 2018.

21. Yi Y, Zhao Y, Li C, Zhang L, Huang H, Li Y, Liu L, Hou P, Cui T, Tan P, et al: RAID v2.0: An updated resource of RNA-associated interactions across organisms. Nucleic Acids Res 45 (D1): D115-D118, 2017.

22. Piñero J, Queralt-Rosinach N, Bravo À, Deu-Pons J, Bauer-Mehren A, Baron M, Sanz F and Furlong LI: DisGeNET: A discovery platform for the dynamical exploration of human diseases and their genes. Database (Oxford) 2015: bav028, 2015.

23. Cho S, Jang I, Jun Y, Yoon S, Ko M, Kwon Y, Choi I, Chang H, Ryu D, Lee B, et al: MiRGator v3.0: A microRNA portal for deep sequencing, expression profiling and mRNA targeting. Nucleic Acids Res 41 (D1): D252-D257, 2013.

24. Wilkerson MD and Hayes DN: ConsensusClusterPlus: A class discovery tool with confidence assessments and item tracking. Bioinformatics 26: 1572-1573, 2010.

25. Kuleshov MV, Jones MR, Rouillard AD, Fernandez NF, Duan Q, Wang Z, Koplev S, Jenkins SL, Jagodnik KM, Lachmann A, et al: Enrichr: a comprehensive gene set enrichment analysis web server 2016 update. Nucleic Acids Res 44: W90-W97, 2016.

26. Amaral LA, Scala A, Barthelemy M and Stanley HE: Classes of small-world networks. Proc Natl Acad Sci USA 97: 11149-11152, 2000.

27. Simos T, Georgopoulou U, Thyphronitis G, Koskinas J and Papaloukas C: Analysis of protein interaction networks for the detection of candidate hepatitis $\mathrm{B}$ and $\mathrm{C}$ biomarkers. IEEE J Biomed Health Inform 19: 181-189, 2015.

28. Tawfeek MA, Alfadhli EM, Alayoubi AM, El-Beshbishy HA and Habib FA: Sex hormone binding globulin as a valuable biochemical marker in predicting gestational diabetes mellitus. BMC Womens Health 17: 18, 2017.

29. Kramer CK, Swaminathan B, Hanley AJ, Connelly PW, Sermer M, Zinman B and Retnakaran R: Vitamin D and parathyroid hormone status in pregnancy: Effect on insulin sensitivity, $\beta$-cell function, and gestational diabetes mellitus J Clin Endocrinol Metab 99: 4506-4513, 2014.

30. Serra MC and Ryan AS: Influence of Vitamin D and parathyroid hormone on bone and metabolic risk in women with previous gestational diabetes. Horm Metab Res 48: 497-502, 2016.

31. Khan S and Jena GB: Protective role of sodium butyrate, a HDAC inhibitor on beta-cell proliferation, function and glucose homeostasis through modulation of p38/ERK MAPK and apoptotic pathways: Study in juvenile diabetic rat. Chem Biol Interact 213 : $1-12,2014$

32. Lee P, Linderman JD, Smith S, Brychta RJ, Wang J, Idelson C, Perron RM, Werner CD, Phan GQ, Kammula US, et al: Irisin and FGF21 are cold-induced endocrine activators of brown fat function in humans. Cell Metab 19: 302-309, 2014.

33. Zhang Y, Li R, Meng Y, Li S, Donelan W, Zhao Y, Qi L, Zhang M, Wang X, Cui T, et al: Irisin stimulates browning of white adipocytes through mitogen-activated protein kinase p38 MAP kinase and ERK MAP kinase signaling. Diabetes 63: 514-525, 2014

34. Zhang Y, Mu Q, Zhou Z, Song H, Zhang Y, Wu F, Jiang M, Wang F, Zhang W, Li L, et al: Protective effect of irisin on atherosclerosis via suppressing oxidized low density lipoprotein induced vascular inflammation and endothelial dysfunction. PLoS One 11: e0158038, 2016.
35. Pan D, Mao C, Quattrochi B, Friedline RH, Zhu LJ, Jung DY, Kim JK, Lewis B and Wang YX: MicroRNA-378 controls classical brown fat expansion to counteract obesity. Nat Commun 5: 4725, 2014.

36. Samy DM, Ismail CA and Nassra RA: Circulating irisin concentrations in rat models of thyroid dysfunction - effect of exercise. Metabolism 64: 804-813, 2015.

37. Tian L, He Y, Zhang H, Wu Z, Li D and Zheng C: Comprehensive analysis of differentially expressed profiles of lncRNAs and mRNAs reveals ceRNA networks in the transformation of diffuse large B-cell lymphoma. Oncol Lett 16: 882-890, 2018.

38. Liu H, Zhang Z, Wu N, Guo H, Zhang H, Fan D, Nie Y and Liu Y: Integrative Analysis of Dysregulated IncRNA-Associated ceRNA Network Reveals Functional lncRNAs in Gastric Cancer. Genes (Basel) 9: 9, 2018.

39. Feng K, Liu Y, Xu LJ, Zhao LF, Jia CW and Xu MY: Long noncoding RNA PVT1 enhances the viability and invasion of papillary thyroid carcinoma cells by functioning as ceRNA of microRNA-30a through mediating expression of insulin like growth factor 1 receptor. Biomed Pharmacother 104: 686-698, 2018.

40. Lin Z, Li X, Zhan X, Sun L, Gao J, Cao Y and Qiu H: Construction of competitive endogenous RNA network reveals regulatory role of long non-coding RNAs in type 2 diabetes mellitus. J Cell Mol Med 21: 3204-3213, 2017.

41. Zhang C, Yang X, Qi Q, Gao Y, Wei Q and Han S: IncRNA-HEIH in serum and exosomes as a potential biomarker in the HCV-related hepatocellular carcinoma. Cancer Biomark 21: 651-659, 2018.

42. Yin Q, Wu A and Liu M: Plasma long non-coding RNA (lncRNA) GAS5 is a new biomarker for coronary artery disease. Med Sci Monit 23: 6042-6048, 2017.

43. Cao Y, Wang P, Ning S, Xiao W, Xiao B and Li X: Identification of prognostic biomarkers in glioblastoma using a long non-coding RNA-mediated, competitive endogenous RNA network. Oncotarget 7: 41737-41747, 2016.

44. Kadiyala R, Peter R and Okosieme OE: Thyroid dysfunction in patients with diabetes: Clinical implications and screening strategies. Int J Clin Pract 64: 1130-1139, 2010.

45. Lambadiari V, Mitrou P, Maratou E, Raptis AE, Tountas N, Raptis SA and Dimitriadis G: Thyroid hormones are positively associated with insulin resistance early in the development of type 2 diabetes. Endocrine 39: 28-32, 2011.

46. Vondra K, Vrbikova J and Dvorakova K: Thyroid gland diseases in adult patients with diabetes mellitus. Minerva Endocrinol 30: 217-236, 2005.

47. Li WD, Jiao H, Wang K, Yang F, Grant SF, Hakonarson H, Ahima R and Arlen Price R: Pathway-based genome-wide association studies reveal that the racl pathway is associated with plasma adiponectin levels. Sci Rep 5: 13422, 2015.

48. Moreno-Navarrete JM, Ortega F, Serrano M, Guerra E, Pardo G, Tinahones F, Ricart $\mathrm{W}$ and Fernández-Real JM: Irisin is expressed and produced by human muscle and adipose tissue in association with obesity and insulin resistance. J Clin Endocrinol Metab 98: E769-E778, 2013.

This work is licensed under a Creative Commons Attribution-NonCommercial-NoDerivatives 4.0 International (CC BY-NC-ND 4.0) License. 